\title{
Impurity states in the quantum spin Hall phase in graphene
}

\author{
J. W. González ${ }^{1}$ and J. Fernández-Rossier ${ }^{1,2}$ \\ ${ }^{1}$ International Iberian Nanotechnology Laboratory - INL, Av. Mestre José Veiga, 4715-330 Braga, Portugal \\ ${ }^{2}$ Departamento de Física Aplicada, Universidad de Alicante, 03690 Alicante, Spain
}

(Received 16 May 2012; published 21 September 2012)

\begin{abstract}
Two-dimensional insulators with time-reversal symmetry can have two topologically different phases, the quantum spin Hall and the normal phase. The former is revealed by the existence of conducting edge states that are topologically protected. Here we show that the reaction to impurity, in bulk, is radically different in the two phases and can be used as a marker for the topological phase. Within the context of the Kane-Mele model for graphene, we find that strictly normalizable in-gap impurity states only occur in the quantum spin Hall phase and carry a dissipationless current whose chirality is determined by the spin and pseudospin of the residing electron.
\end{abstract}

DOI: 10.1103/PhysRevB.86.115327

PACS number(s): 73.20.Hb, 73.20.At, 73.43.-f

\section{INTRODUCTION}

The intrinsic properties of an electron gas are revealed in the way it reacts to the presence of a localized perturbation. In metals, the period of Friedel oscillations provides information about the Fermi surface. ${ }^{1}$ In semiconductors, the binding energy of shallow acceptors and donor states depends on the effective mass and dielectric constant of the host material. ${ }^{2}$ In superconductors, both the impurity induced modulations of the density of states ${ }^{3}$ and the presence of zero energy or midgap states, ${ }^{4}$ reveal the symmetry of their order parameter. Here we address the fundamental question of whether two-dimensional topological insulators react to a localized perturbation in a way different from conventional insulators.

Topological insulators ${ }^{5}$ have a bulk band gap like a normal insulator but have protected conducting states on their edges and surfaces. This bulk-boundary correspondence has been rigorously established ${ }^{6,7}$ in boundaries that preserve the translational invariance in at least one dimension. In twodimensional topological insulators, edge state are expected to present quantized conductance ${ }^{8-11}$ which can be used to unveil the existence of bulk topological order. ${ }^{12}$ More recently, the bulk-boundary correspondence has been extended to the case of topological defects that lead to Hamiltonians $\mathcal{H}(\mathbf{k}, \mathbf{r})$ that vary slowly with adiabatic parameters $\mathbf{r}$ surrounding the defect. $^{13,14}$

Here we explore the electronic structure of a twodimensional insulator, that can be either in the quantum spin Hall (QSH) or in the normal phase, in the neighborhood of an isoelectronic impurity that creates a repulsive short-range potential. We find that only in the QSH phase in-gap states appear and have exotic electronic properties: they carry nondissipative spin current. Our finding provides an alternative way to detect topological order, using local probes sensitive to the density of states in the neighborhood of the impurities.

We use gapped graphene as a toy model for twodimensional topological insulators. ${ }^{8,9}$ The electronic properties of graphene are intimately related to the structure of the honeycomb lattice, formed by two interpenetrating triangular sublattices, $A$ and $B$, related by inversion symmetry, which define a pseudospin degree of freedom that we denote with the operator $\tau_{z}$. There are two ways to open a gap in graphene preserving the size of its two atom minimal unit cell. Both lead to interesting electronic phases. A conventional gap opens in graphene when a sublattice symmetry breaking potential, $\frac{\Delta_{0}}{2} \tau_{z}$, is included in the Hamiltonian. This gap entails peculiar electronic properties: the two valleys carry orbital currents of opposite sign. ${ }^{15}$ When the gap is opened by intrinsic spin orbit coupling, as described with the second-neighbor spin-dependent hopping proposed by Kane-Mele, graphene is in the QSH phase.

The rest of this paper is organized as follows. In Sec. II we review the Kane-Mele model for gapped graphene without impurities. In Sec. III we study the appearance of in-gap impurity states due to a localized repulsive potential. We consider both the full lattice description, as well as the continuum limit and we discuss the properties of the wave function of the in-gap state. In Sec. IV we show that, associated to the in-gap state, there is a spin current with two contributions, one coming from the in-gap state and the other coming from the perturbed band states. Our conclusions are presented in Sec. V.

\section{THE MODEL}

The Kane-Mele ${ }^{8,9}$ tight-binding Hamiltonian $\mathcal{H}_{0}$ describes electrons in a hexagonal lattice, with first neighbor hopping $t$, spin-dependent second neighbor hopping $t_{s o}$ and the $\frac{\Delta_{0}}{2} \tau_{z}$ term. This model commutes with $S_{z}$, the spin projection perpendicular to the graphene plane, and each spin sector is identical to the Haldane model ${ }^{16}$ for spinless fermions. We consider the effect of a substitutional isoelectronic impurity, described with a single-site potential, in an otherwise boundless and perfect gapped system:

$$
\mathcal{H}=\mathcal{H}_{0}+V_{0} \sum_{s}|0 s\rangle\langle 0 s| .
$$

The strength of the spin-independent impurity potential is $V_{0}$, and acts only on the atom 0 of the $A$ sublattice.

For each spin channel $s$, the crystal Hamiltonian $\mathcal{H}_{0}$ can be written in the form of a $2 \times 2$ matrix in the sublattice basis:

$$
\mathcal{H}_{\mathbf{k}}^{s}=t\left[f(\mathbf{k}) \tau^{+}+f^{*}(\mathbf{k}) \tau^{-}\right]+\frac{\Delta_{0}}{2} \tau_{z}+s t_{s o} g(\mathbf{k}) \tau_{z},
$$

where $\vec{\tau}=\left(\tau_{x}, \tau_{y}, \tau_{z}\right)$ are the Pauli matrices in the sublattice space, $\tau^{ \pm}=\tau_{x} \pm i \tau_{y}, s= \pm 1$ are the eigenvalues of the spin operator $S_{z} \cdot f(\mathbf{k})$ and $g(\mathbf{k})$ are the usual functions that sum the 

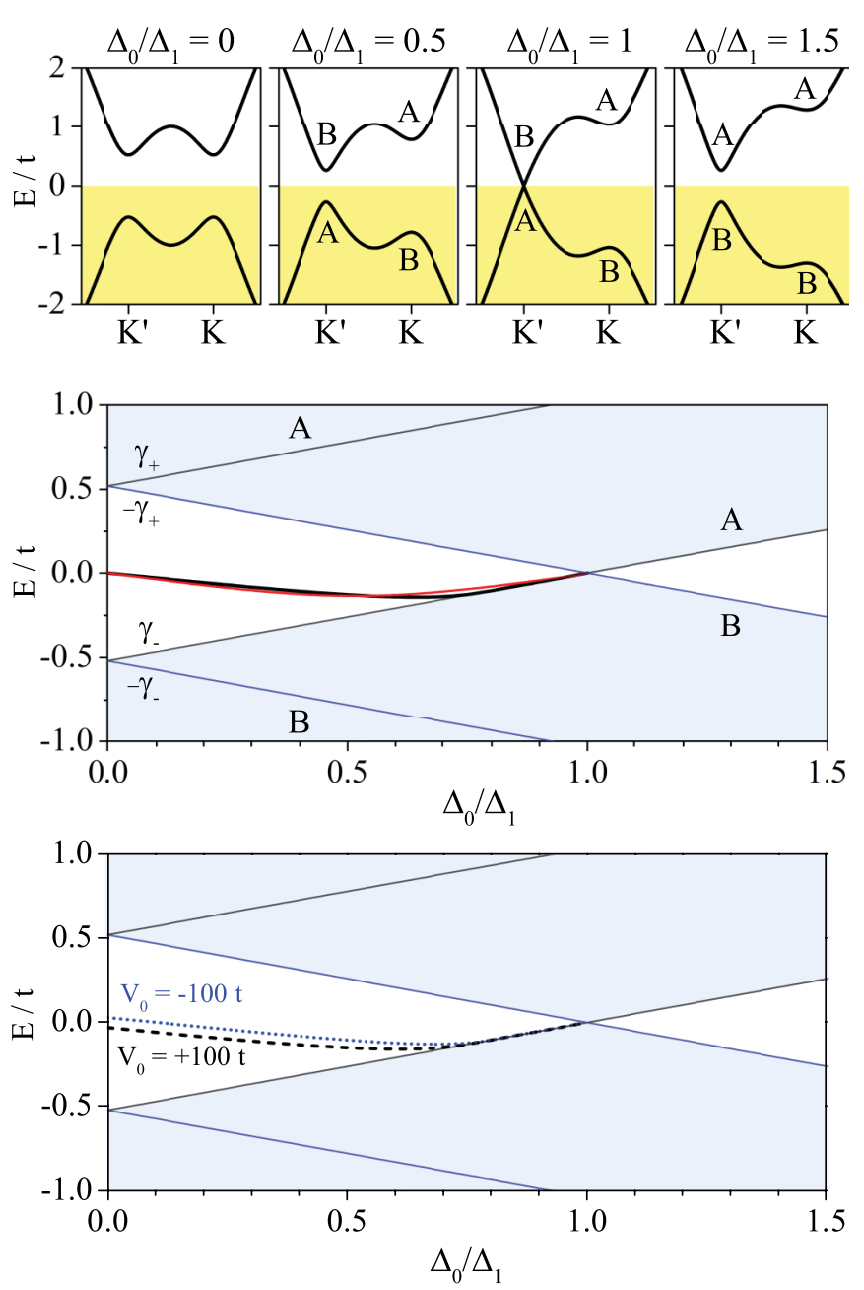

FIG. 1. (Color online) Top panel: energy bands for the $s=+$ spin channel, with $t_{s o}=0.1 t$, and four different values of $\Delta_{0}$. Middle panel: evolution of bands at $K$ and $K^{\prime}$ and in-gap impurity energy $E_{b}\left(\Delta_{0}\right)$ calculated both with the lattice model (black) and the continuum model (red). Bottom panel: in-gap impurity energy $E_{b}\left(\Delta_{0}\right)$ for a repulsive potential $V_{0}=100 t$ (dashed line) and for a attractive potential $V_{0}=-100 t$ (dotted line).

Bloch phase over the nearest and path-dependent next-nearest neighbors. ${ }^{9}$

The Bloch Hamiltonian is identical to that of a (pseudo)spin $\vec{\tau}$ in an effective field: $\mathcal{H}_{\mathbf{k}}^{s}=\vec{h}_{\mathbf{k}}^{s} \cdot \vec{\tau}$, where

$$
\vec{h}_{\mathbf{k}}^{s}=\left[t f(\mathbf{k})+t f^{*}(\mathbf{k}), t f(\mathbf{k})-t f^{*}(\mathbf{k}), \frac{\Delta_{0}+t_{s o} g(\mathbf{k})}{2}\right] .
$$

The energy bands for the model are given by $\epsilon_{\nu, s}(\mathbf{k})= \pm \epsilon_{\mathbf{k}}^{s}$, where $v= \pm$ labels the two bands (per spin channel) and $\epsilon_{\mathbf{k}}^{s} \equiv$ $\left|\vec{h}_{\mathbf{k}}^{s}\right|$. In the top panel of Fig. 1 we plot them for a given value of $s$, along the line that joins the Dirac points $K$ and $K^{\prime}$, all of them with the same values of $t$ and $t_{s o}=0.1 t$ and different values of $\Delta_{0}$. Time-reversal invariance ensures that, for the opposite spin orientations, we have $\epsilon_{v, s}(\mathbf{k})=\epsilon_{\nu, \bar{s}}(-\mathbf{k})$. The $K$ and $K^{\prime}$ points define the so-called valley index $\sigma= \pm 1$. These points are special because the in-plane components of the effective field $\vec{h}_{\mathbf{k}}^{s}$ vanish, making the energy splitting between the bands minimal. At $K$ and $K^{\prime}$ we have $\vec{h}_{\mathbf{k}}^{s}=\left(0,0, \gamma_{s \sigma}\right)$ with

$$
\gamma_{s \sigma}=\frac{1}{2}\left(\Delta_{0}+s \sigma \Delta_{1}\right)
$$

where $s \sigma$ can take only two values $s \sigma= \pm 1$ and $\Delta_{1}=6 \sqrt{3} t_{s o}$. Thus, at the Dirac point the wave functions have a well defined sublattice $\left(\tau_{z}\right)$ character. The effective field at the Dirac points, $\pm \gamma_{s \sigma}$, defines the top of the valence band and bottom of the conduction band at each valley. We plot them in the low panel of Fig. 1.

In the transition from the QSH phase, with $\gamma_{-}<0$ to the normal phase, with $\gamma_{-}>0$, the system closes the gap $\left(\gamma_{-}=\right.$ 0 ), reflecting the impossibility of deforming adiabatically one phase into the other. ${ }^{8,9}$ In the QSH phase, the energy splitting is finite at both valleys, the orientation of the effective field $\vec{h}_{\mathbf{k}}^{s}$ is opposite at $K$ and $K^{\prime}$, for a given $s$. In this phase, the model presents topologically protected edge states inside the gap, making the edge metallic. At the normal phase, with $\gamma_{-}>0$ the orientation of the effective field $\vec{h}_{\mathbf{k}}^{s}$ is now the same at both valleys and a gap opens in the edge states.

\section{IN-GAP STATES}

We now explore the possible appearance of in-gap states in the two insulating phases of the model in the presence of the impurity potential, as described by Eq. (1). This is different from previous works that have studied the influence of impurities on the conducting surface states of 3D topological insulators. ${ }^{17,18} \mathrm{We}$ address our problem using the $T$-matrix formalism, ${ }^{19}$ which has been widely used in the context of graphene. ${ }^{20-25}$ We define the Green function operators, $\mathcal{G}(z) \equiv$ $(z \mathbb{I}-\mathcal{H})^{-1}$, and $\mathcal{G}_{0}(z) \equiv\left(z \mathbb{I}-\mathcal{H}_{0}\right)^{-1}$, where $z$ is a complex number and $\mathbb{I}$ is the unit matrix in the Hilbert space of the infinite lattice. A closed expression for the Green function $\mathcal{G}_{0}(z)$ can be readily obtained in terms of the eigenstates of $\mathcal{H}_{\mathbf{k}}^{s}$. For the on-site spin-independent potential, the complete Green function is related to $\mathcal{G}_{0}(z)$ by

$$
\mathcal{G}(z)=\mathcal{G}^{0}(z)+\mathcal{G}^{0}(z)|0\rangle \frac{V_{0}}{1-V_{0} \mathcal{G}_{0,0}^{0}(z)}\langle 0| \mathcal{G}^{0}(z),
$$

where $\mathcal{G}_{0,0}^{0}(z)$ is the unperturbed Green function diagonal matrix element in the atomic representation and 0 is the atom at which the perturbation is located.

The appearance of bound states with energy $E_{b}$ would be given by the existence of poles of $\mathcal{G}$ in the band gap defined by $\mathcal{H}_{\mathbf{k}}^{s}$. Thus, we have to solve the equation

$$
\mathcal{G}_{0,0}^{0}\left(E_{b}\right)=\frac{1}{V_{0}} \text {. }
$$

\section{A. Lattice model}

In order to have a closed expression for $\mathcal{G}_{0,0}^{0}\left(E_{b}\right)$ we use the Lehman representation and project over the site representation. Any given site in the lattice $i$ can be identified by its unit cell $\vec{R}_{i}$ and the sublattice $\tau_{i}=A, B$. The unperturbed Green function is written in terms of the eigenstates of the crystal Hamiltonian

$$
|\nu, \mathbf{k}, s\rangle=\frac{1}{\sqrt{N}} \sum_{\vec{R}, \tau} e^{i \mathbf{k} \cdot \mathbf{R}} \mathcal{U}_{\nu, \mathbf{k}, \tau}^{s}|\vec{R} \tau, s\rangle,
$$


where $N$ is the number of unit cells of the crystal, and $\mathcal{U}_{v, \mathbf{k}, \tau}^{s}$ are the components of the eigenstates of the Bloch Hamiltonian in Eq. (2). If we express the effective field in spherical coordinates as, $\vec{h}_{\mathbf{k}}^{s}=\epsilon_{\mathbf{k}}^{s}\left(\sin \theta_{\mathbf{k}}^{s} \cos \varphi_{\mathbf{k}}^{s}, \sin \theta_{\mathbf{k}}^{s} \sin \varphi_{\mathbf{k}}^{s}, \cos \theta_{\mathbf{k}}^{s}\right)$, the corresponding wave functions for the $v= \pm$ bands read

$$
\begin{aligned}
& \mathcal{U}_{-, \mathbf{k}, A}^{s}=\sin \left(\frac{\theta}{2}\right) e^{-i \varphi}, \quad \mathcal{U}_{-, \mathbf{k}, B}^{s}=-\cos \left(\frac{\theta}{2}\right), \\
& \mathcal{U}_{+, \mathbf{k}, A}^{s}=\cos \left(\frac{\theta}{2}\right) e^{-i \varphi}, \quad \mathcal{U}_{+, \mathbf{k}, B}^{s}=\sin \left(\frac{\theta}{2}\right),
\end{aligned}
$$

where we have omitted the subscripts from $\theta$ and $\varphi$ for the sake of clarity. The unperturbed Green function matrix can be written as

$$
\mathcal{G}_{i j}^{0}(z)=\frac{1}{N} \sum_{\mathbf{k}, v} \frac{e^{\mathbf{k} \cdot\left(\mathbf{R}_{i}-\mathbf{R}_{\mathbf{j}}\right)}}{z-\varepsilon_{\mathbf{k}, v}}\left(\mathcal{U}_{v, \mathbf{k}, \tau_{i}}^{s}\right)^{*} \mathcal{U}_{\nu, \mathbf{k}, \tau_{j}}^{s}
$$

Using this expression in combination with Eq. (6), we can recast Eq. (5) as

$$
\frac{1}{V_{0}}=\frac{1}{2 N} \sum_{\mathbf{k}}\left[\frac{1-\vec{n}_{\mathbf{k}}^{s} \cdot \hat{z}}{E_{b}+\epsilon_{\mathbf{k}}^{s}}+\frac{1+\vec{n}_{\mathbf{k}}^{s} \cdot \hat{z}}{E_{b}-\epsilon_{\mathbf{k}}^{s}}\right],
$$

where $\vec{n}_{\mathbf{k}}^{s}=\vec{h}_{\mathbf{k}}^{s} / \epsilon_{\mathbf{k}}^{s}$. Importantly, the Green function $\mathcal{G}_{00}^{0}(z)$ is expressed as a sum over the whole Brillouin zone of a function that depends on the projection of the effective field vector and, as such, it contains information of the topology of the Bloch states, which is a necessary condition to expect a relation between the solutions of Eq. (8) and the topological order in the system.

Equation (8) can be analytically solved in the strong coupling limit $V_{0}^{-1}=0$, in two cases. For $\Delta_{0}=0$, the repulsive potential yields a midgap state $E_{b}=0$ whose properties we discuss below. For $\Delta_{1}=0$, there is a solution with $E_{b}=$ $-\Delta_{0} / 2$, in agreement with a general result. ${ }^{26}$ However, this solution is degenerate with the top of the valence band and it is a resonance rather than an actual in-gap state. The interpolation between these two limits is obtained by the numerical solution of Eq. (8) and is shown in the middle panel of Fig. 1 for $V_{0}=10^{6} t$. Bound states are only found when $V_{0}>t$ and, interestingly, only when $\Delta_{0}<\Delta_{1}$, i.e., in the topological insulator phase. This is the main result of the paper: we find that a local impurity can bind an in-gap state only in the QSH phase.

The effect of the sign of the impurity potential is shown in the bottom panel of Fig. 1. Depending on the sign of the impurity potential, the in-gap state at $\Delta_{0}=0$ occurs at an energy $\mp E_{b}$. Even more importantly, the trend of the in-gap impurity energy with $\Delta_{0} / \Delta_{1}$ does not change with the sign of $V_{0}$. Again, the in-gap state only appears in the QSH phase.

\section{B. Continuum limit}

In order to obtain some analytical insight of the one to one relation between the existence of in-gap states and the topological phase, we have worked out Eq. (7) in the continuum limit, in which only states close to the two Dirac points are included. Their crystal Hamiltonian is then given by

$$
\mathcal{H}_{0}(\vec{k}, \sigma, s)=\hbar v_{F}\left(k_{x} \tau_{x}+\sigma k_{y} \tau_{y}\right)+\frac{\Delta_{0}}{2} \tau_{z}+\frac{\Delta_{1}}{2} s \sigma \tau_{z} .
$$

For this model it is possible to obtain a closed analytical expression for Eq. (8):

$$
\frac{1}{V_{0}}=\frac{a^{2}}{2 \pi\left(\hbar v_{F}\right)^{2}} \sum_{s \tau= \pm}\left(\gamma_{s \tau}-E_{b}\right) \log \left[1+\frac{\varepsilon_{c}^{2}}{\gamma_{s \tau}^{2}-E_{b}^{2}}\right],
$$

where $\epsilon_{c}$ is the cutoff energy. ${ }^{27}$ The sum over $s \tau$ reflects, for a given spin orientation, the contributions coming from both valleys. In the strong coupling limit, $V_{0}^{-1}$ is negligible and the existence of an in-gap solution of Eq. (10) requires that the right hand side sum vanishes. Since $\gamma_{+}-E_{b}$ is always positive, $E_{b}$ must satisfy $\gamma_{-}<E_{b}$, which, by definition of in-gap state, is only satisfied in the QSH phase and not in the normal phase (see lower panel of Fig. 1). Thus, the continuum model also has the one on one relation between topological order and the emergence of in-gap impurity states. The numerical solution of Eq. (10) is shown in Fig. 1 for $V_{0}^{-1}=0$. The energy cut-off $\epsilon_{c}=0.3 t$ was chosen to fit the lattice model results. In general, good agreement between the two models should only occur for extended in-gap states, which occur $E_{b}$ close to one of the bands.

\section{IMPURITY SPIN CURRENT}

In this section we calculate the spin current associated to the presence of the impurity potential. The current is given by the contributions of all occupied states, that includes both the in-gap state and the valence band states. For the former it is possible to obtain a simple closed expression of its wave function, which permits a straightforward calculation of the current. For the band state make use of the Green function formalism, which permits an exact evaluation of the current induced by the impurity.

\section{A. In-gap state wave function}

The Green's function formalism provides a closed expression for the in-gap wave function in terms of their binding energy $E_{b}$, obtained from Eq. (7), and the unperturbed Green function: ${ }^{19}$

$$
\left|\phi_{b}\right\rangle=\left(-\frac{d \mathcal{G}_{00}^{0}\left(E_{b}\right)}{d E}\right)^{-\frac{1}{2}} \sum_{i} \mathcal{G}_{i, 0}^{0}\left(E_{b}\right)|i\rangle .
$$

We consider the case $\Delta_{0}=0$ and $V_{0}^{-1}=10^{-6} t$. Expectedly, the in-gap wave function is localized around the impurity site, as shown in Fig. 2. Interestingly, the in-gap states is not fully sublattice polarized, in contrast with zero energy states in bipartite Hamiltonians. ${ }^{28}$ Thus, we have $\left\langle\phi_{b}\left|\sigma_{z}\right| \phi_{b}\right\rangle \simeq 0.56$, significantly below 1 . We also find, analytically, that the wave function in the impurity sublattice is purely imaginary, while for the other sublattice is purely real. In the reciprocal space it is also true that the wave function has unequal weight on both valleys, but is not fully valley polarized. Both the incomplete sublattice and valley polarizations are quantitatively different from spin-filter edge states in zigzag ribbons for the same model, which are fully sublattice and valley polarized. In contrast, the most salient feature of the edge states is also present for the topological in-gap impurity states: they carry current, which is quite unusual for a localized state. 


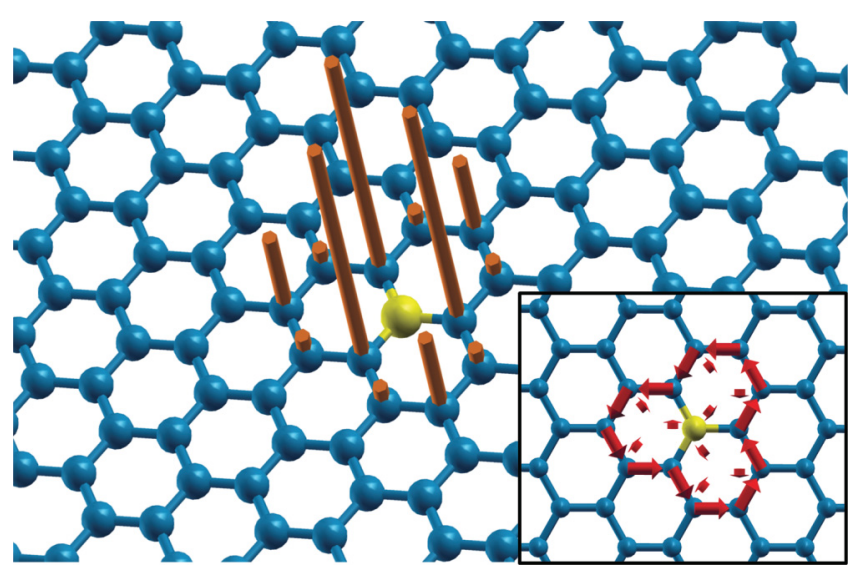

FIG. 2. (Color online) Local density of states as a bar in a exponential scale. The in-set shows the impurity-induced current between links for the state at $E_{b}$. The impurity site is shown in yellow.

\section{B. Calculation of the current}

The current operator is defined at the bonds of the tightbinding Hamiltonian imposing the continuity equation. For a given pair of sites $n$ and $m$ in the lattice, the current operator $\operatorname{reads}^{29} \hat{J}_{n m}=j_{n m}|n\rangle\left\langle m\left|-j_{m n}\right| m\right\rangle\langle n|$, where $j_{m n}=e t_{n m} / i \hbar$ and $t_{n m}$ is the Hamiltonian matrix element $\left\langle n\left|\mathcal{H}_{0}\right| m\right\rangle$. Thus, the current carried by bound states reads

$$
I_{n m}^{\text {bound }}=\frac{e}{\hbar} \operatorname{Im}\left[t_{n m} \phi_{b}^{*}(m) \phi_{b}(n)\right] .
$$

The map of the current is shown, for a given spin, in the inset of Fig. 2, for $t_{s o}=0.1 t, \Delta_{0}=0, V_{0}=10^{6} t$. Although the in-gap state is more localized in the $B$ sublattice, opposite to the impurity site, the current is larger in the $A B$ bonds than in the $B B$ bonds because $t=10 t_{s o}$. For the opposite spin, the current flow changes sign so that the net current is zero but the spin-current is not. We have also verified that, when the impurity site is in the other sublattice, the current flow is inverted. Thus, the in-gap states have dissipationless spin-currents whose chirality is determined by the sublattice at which the impurity resides.

The observable current probed experimentally would be given by the contribution of all occupied states, which includes both the in-gap state and the valence band, whose density of states are shown in the upper inset of Fig. 3. The contribution to the current from the band states reads

$$
I_{n m}^{\text {band }} \equiv \frac{-1}{\pi} \int_{-\infty}^{\gamma_{-}} \operatorname{Im}\left[\mathcal{G}_{n m}(E) j_{m n}-\mathcal{G}_{m n}(E) j_{n m}\right] \mathrm{d} E,
$$

where $\mathcal{G}$ is the full Green function whose closed form is given in Eq. (4). Thus, there are two contributions to the current, one given by the band states and the other given by the in-gap states. We plot both them in Fig. 3, for $\Delta_{0}=0$, as a function of the impurity strength, $V_{0}$. They have opposite signs and, in the strong $V_{0}$ limit, cancel each other. The evolution of $E_{b}\left(V_{0}\right)$ is shown in the lower inset. In that limit the impurity bound state becomes a midgap state $E_{b}=0$ and the spectrum recovers electron-hole symmetry, for which ground state currents are not possible. ${ }^{30}$ The cancellation of the edge current due to the contribution of the bulk states takes also place in the case of

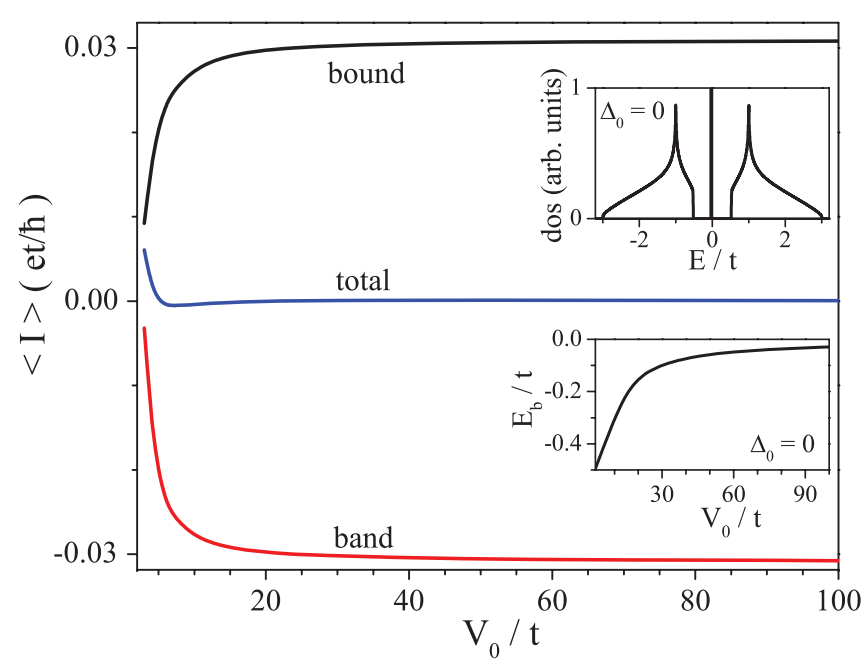

FIG. 3. (Color online) Bound, band and total current in an $A B$ bond, as a function of $V_{0}$. Upper inset: density of states on $B$ sublattice. Lower inset: evolution of $E_{b}\left(V_{0}\right)$ for $\Delta_{0}=0$.

ribbons. However, a finite nonzero spin-current is obtained in our case for a wide range of $V_{0}$ close to what it is expected for absorption of atomic hydrogen in graphene.

\section{DISCUSSION AND CONCLUSIONS}

We now connect our results with previous work on related systems. Although they are superficially similar to the mid gap states of a domain wall of polyacetylene, ${ }^{31}$ the impurity in-gap states do not give rise to charge fractionalization. This can be understood as follows. Together with the in-gap solution of Eq. (5) where $E_{b} \simeq V_{0}^{-1}$, there is always a second solution with energy $E_{b} \simeq V_{0}$. Thus, there are two states outside of the bands, and not only one as in the case of Su-Schrieffer-Heeger solitons, ${ }^{31}$ so that both bands lose a complete state.

Recent theory work has shown that vacancies induce in-gap states both in a different model for a two-dimensional topological insulator, ${ }^{32}$ and for the Kane-Mele-Fu ${ }^{33}$ model for a three-dimensional topological insulator. ${ }^{34}$ However, in-gap impurity states can be found in graphene-based conventional insulators, like one-dimensional armchair graphene ribbons, ${ }^{28}$ and two-dimensional graphane, ${ }^{35}$ although they are not expected to carry spin currents.

In conclusion, we have shown that two-dimensional topological insulators can be distinguished from conventional insulators by the radically different electronic properties of their impurity states. Within the Kane-Mele model, we have shown that in-gap impurity states appear only in the case topologically nontrivial or quantum spin Hall phase $\mathrm{e}^{8,9}$ and not in the normal phase. These topological in-gap impurity states carry a net spin-current which, due to the single occupancy of the state, is upgraded to a net current or orbital magnetization. Therefore, we propose that the topological nature of this type of insulators can be established by local probes of the electronic properties of these defects, as opposed to the highly nonlocal probes required to test quantized transport of the edge states. 


\section{ACKNOWLEDGMENTS}

This work has been financially supported by MEC-Spain (Grant Nos. FIS2010-21883-C02-01, FIS2009-08744, and
CONSOLIDER CSD2007-0010) as well as Generalitat Valenciana, grant no. Prometeo 2011-12. We are indebted to A. Balatsky, A. M. Black-Schaffer, and F. Delgado for fruitful discussions.
${ }^{1}$ M. F. Crommie, C. P. Lutz, D. M. Eigler, Nature (London) 363, 524 (1993); P. T. Sprunger, L. Petersen, E. W. Plummer, E. Laegsgaard, and F. Besenbacher, Science 275, 1764 (1997).

${ }^{2}$ G. L. Pearson and J. Bardeen, Phys. Rev. 75, 865 (1949).

${ }^{3}$ J. M. Byers, M. E. Flatté, and D. J. Scalapino, Phys. Rev. Lett. 71, 3363 (1993); M. I. Salkola, A. V. Balatsky, and D. J. Scalapino, ibid. 77, 1841 (1996)

${ }^{4}$ Chia-Ren Hu, Phys. Rev. Lett. 72, 1526 (1994).

${ }^{5}$ M. Z. Hasan and C. L. Kane, Rev. Mod. Phys. 82, 3045 (2010).

${ }^{6}$ B. I. Halperin, Phys. Rev. B 25, 2185 (1982).

${ }^{7}$ B. Bernevig, T. Hughes, and S. C. Zhang, Science 314, 1757 (2006).

${ }^{8}$ C. L. Kane and E. J. Mele, Phys. Rev. Lett. 95, 226801 (2005).

${ }^{9}$ C. L. Kane and E. J. Mele, Phys. Rev. Lett. 95, 146802 (2005).

${ }^{10}$ S. Murakami, J. Phys.: Conf. Ser. 302, 012019 (2011).

${ }^{11}$ D. Gosalbez, D. Soriano, J. J. Palacios, and J. Fernández-Rossier, Solid State Commun. 152, 1469 (2012).

${ }^{12}$ M. Konig, S. Wiedmann, C. Brüne, A. Roth, H. Buhmann, L. W. Molenkamp, X. L. Qi, and S. C. Zhang, Science 318, 766 (2007).

${ }^{13}$ J. C. Y. Teo and C. L. Kane, Phys. Rev. B 82, 115120 (2010).

${ }^{14}$ M. M. Ugeda, I. Brihuega, F. Guinea, and J. M. Gómez-Rodríguez, Phys. Rev. Lett. 104, 096804 (2010).

${ }^{15}$ D. Xiao, W. Yao, and Q. Niu, Phys. Rev. Lett. 99, 236809 (2007).

${ }^{16}$ F. D. M. Haldane, Phys. Rev. Lett. 61, 2015 (1988).

${ }^{17}$ R. R. Biswas and A. V. Balatsky, Phys. Rev. B 81, 233405 (2010).

${ }^{18}$ A. M. Black-Schaffer and A. V. Balatsky, Phys. Rev. B 85, 121103 (2012).

${ }^{19}$ E. Economou, Green's Functions in Quantum Physics (SpringerVerlag, Berlin, 1979).

${ }^{20}$ V. M. Pereira, J. M. B. Lopes dos Santos, and A. H. Castro Neto, Phys. Rev. B 77, 115109 (2008).
${ }^{21}$ V. M. Pereira, F. Guinea, J. M. B. Lopes dos Santos, N. M. R. Peres, and A. H. Castro Neto, Phys. Rev. Lett. 96, 036801 (2006).

${ }^{22}$ B. R. K. Nanda, M. Sherafati, Z. Popović, and S. Satpathy, New J. Phys. 14, 083004 (2012).

${ }^{23}$ C. Bena, Phys. Rev. B 79, 125427 (2009).

${ }^{24}$ M. Sherafati and S. Satpathy, Phys. Status Solidi B 248, 2056 (2011).

${ }^{25}$ T. O. Wehling, A. V. Balatsky, M. I. Katsnelson, A. I. Lichtenstein, K. Scharnberg, and R. Wiesendanger, Phys. Rev. B 75, 125425 (2007).

${ }^{26}$ D. Soriano and J. Fernández-Rossier, Phys. Rev. B 85, 195433 (2012).

${ }^{27} \mathrm{~A}$ similar expression, ignoring intervalley coupling, has been obtained by M. Inglot and V. K. Dugaev, J. Appl. Phys. 109, 123709 (2011).

${ }^{28}$ J. J. Palacios, J. Fernández-Rossier, and L. Brey, Phys. Rev. B 77, 195428 (2008).

${ }^{29}$ D. Soriano and J. Fernández-Rossier, Phys. Rev. B 82, 161302(R) (2010).

${ }^{30}$ D. Zheng, G. M. Zhang, and C. Wu, Phys. Rev. B 84, 205121 (2011).

${ }^{31}$ W. P. Su, J. R. Schrieffer, and A. J. Heeger, Phys. Rev. Lett. 42, 1698 (1979).

${ }^{32}$ Wen-Yu Shan, Jie Lu, Hai-Zhou Lu, and Shun-Qing Shen, Phys. Rev. B 84, 035307 (2011).

${ }^{33}$ L. Fu, C. L. Kane, and E. J. Mele, Phys. Rev. Lett. 98, 106803 (2007).

${ }^{34}$ A. M. Black-Schaffer and A. V. Balatsky, arXiv:1202.4872 [cond-mat.mes-hall] (2012).

${ }^{35}$ J. Berashevich and T. Chakraborty, Nanotechnology 21, 355201 (2010). 